\title{
Abdominal Displacement Ventilation: An Effective Intervention for Sedation-Induced Hypoxia
}

\author{
Nikhil Meena $^{a} \quad$ Maggie Macchiarella $^{b}$ Jose Diego Caceres ${ }^{a}$ \\ Thaddeus Bartter ${ }^{\mathrm{a}, \mathrm{c}}$ \\ a Division of Pulmonary and Critical Care Medicine, University of Arkansas for Medical \\ Sciences, Little Rock, AR, USA; ${ }^{b}$ Respiratory Care Department, University of Arkansas for \\ Medical Sciences, Little Rock, AR, USA; ' Veterans Administration Healthcare Services, \\ Little Rock, AR, USA
}

\section{What Is It about?}

- We describe abdominal displacement ventilation (ADV), which helps a patient breathe when their respiratory drive is suppressed. The study targets patients who have been sedated. The technique of pressing on their abdomen with the operator's hand to push the diaphragm up into the chest allows for an assisted exhalation, and when the hand is taken away the lung expands from the negative pressure generated.

- We think this could also be boon for the drug overdoses, as it does not require mucosal contact as in mouth-to-mouth respiration. ADV could change how we provide basic life support.

\section{Keywords \\ Bronchoscopy $\cdot$ Hypoxia $\cdot$ Sedation}

\begin{abstract}
Background: Sedation for bronchoscopy at times causes hypoxia. The application of positive pressure ventilation for sedation-induced hypoxia often requires cessation of the bronchoscopy. In contrast, ventilation effected via cyclical abdominal compression, if effective, would allow bronchoscopy to proceed. Initial trials of abdominal displacement ventilation (ADV) proved successful. This report documents extended experience with ADV. Objective: To evaluate and report the efficacy and applicability of ADV in the setting of sedation-induced hypoxia for consecutive patients over an extended interval. Methods: Based upon its initial efficacy, ADV had been incorporated into the standard approach to sedation-induced hypoxia. We retrospectively reviewed all bronchoscopies performed by interventional pulmonary over a 12-month interval. Management and efficacy of every episode of sedation-induced hypoxia were documented. Results: Over the study interval, 893 bronchoscopies had been performed, with sedation-induced hypoxia occurring in 38 (4\%). ADV was possible in 37 of the 38
\end{abstract}


patients. In every case, ADV was effective and allowed completion of the procedure. There were no adverse effects. Conclusion: ADV is a simple, effective, noninvasive approach to sedation-induced hypoxia that effects adequate ventilation and allows safe continuance of procedures.

\section{Introduction}

Sedation is the standard of care for bronchoscopic procedures. Hypoxia is a known complication of sedation, with frequency of hypoxia depending upon how it is defined [1-4]. Hypoxia can at times lead to transient discontinuation of the procedure or to procedure termination in order to provide ventilatory support. A body of historical data has shown that ventilation can be effectively achieved with abdominal displacement techniques [5-7]. We hypothesized that abdominal displacement might provide adequate temporary ventilation during periods of sedation-induced hypoxia. An advantage of this would be that, if effective, the ventilatory intervention would not interfere with bronchoscopy. Initial trials at this institution of abdominal displacement ventilation (ADV) for sedation-induced hypoxia were effective, and ADV was incorporated into standard practice for sedation-induced hypoxemia. This paper represents a retrospective review of all cases of sedation-induced hypoxemia from August of 2017 to July of 2018. Based upon this experience, we suggest that ADV is a simple, effective, noninvasive intervention that may be of particular value for bronchoscopists faced with transient hypoxia during procedures.

\section{Methods}

For this study, all bronchoscopies performed by interventional pulmonary in the bronchoscopy suite from August 1, 2017, to July 31, 2018, were reviewed, and all cases involving sedation-induced hypoxia were selected. Whereas ADV had been newly incorporated into the approach to hypoxia in early 2017, cases had been bookmarked for quality assurance purposes. A request to report the data was submitted to the institutional IRB and was accepted (IRB \#228841). Management and clinical course for all such cases are reported.

All bronchoscopies performed by interventional pulmonary in the bronchoscopy suite are performed with the patient in a semi-recumbent position (20-30 elevation) to minimize any possible aspiration. The bronchoscopist stands at the right side of the patient, with the monitor across the bed on the left side. For all patients over the study interval, sedation consisted of intravenous propofol given in boluses by the attending physician or by a pulmonary fellow. Vitals are monitored by a nurse. A respiratory therapist is present in all cases. The therapist has easy access to the head of the bed should interventions be indicated. The therapist and/or a pulmonary fellow assists with procedures such as lavage and transthoracic needle aspiration. In order to allow ADV, should it be indicated, all patients are advised to wear loose-fitting trousers and belts are removed prior to bronchoscopy. The initial approach to desaturation is a jaw lift. If this is ineffective and desaturation persists for more than thirty seconds, ADV is begun unless there is a contraindication. If ADV is contraindicated or ineffective, positive pressure ventilation is instituted.

\section{Abdominal Displacement Ventilation}

While the proceduralist and the assistant continue the procedure, the nurse or the respiratory therapist uses the palm of one hand to intermittently compress the abdomen in a cephalad 
Meena et al.: Abdominal Displacement Ventilation for Sedation-Induced Hypoxia

Table 1. Study group demographics and procedure characteristics

\begin{tabular}{lc}
\hline Patients, $n$ & 38 \\
Age, years & $57[18-95]$ \\
Male:female & $21: 17$ \\
Height, cm & $172[149-198]$ \\
Weight, kg & $86[39-145]$ \\
Body mass index & $31[15-41]$ \\
Propofol dosing & $785[150-1010]$ \\
Procedure duration, min & $65[35-95]$ \\
Procedure type & \\
$\quad$ EBUS & $28(76)$ \\
$\quad$ Bronchoscopy with dilation & $8(21)$ \\
$\quad$ Airway survey & $1(2)$ \\
Intervention (ADV:positive pressure & $37: 1$ \\
$\quad$ ventilation) & \\
Minutes in recovery room & $34[29-37]$ \\
Chronic obstructive lung disease & $13(34)$ \\
Restrictive lung disease & $1(2)$ \\
Pleural effusion & $4(10)$ \\
Neuromuscular disease & 0 \\
Obstructive sleep apnea & $9(23)$ \\
Obesity hypoventilation syndrome & $5(13)$ \\
\hline
\end{tabular}

Data are presented as $n(\%)$ or mean [range]. EBUS, endobronchial ultrasound procedures.

direction. Compression effects exhalation via diaphragmatic elevation. Release of pressure leads to a passive negative pressure inhalation. Abdominal compressions are performed at a rate of approximately 100 per min. The maneuver is held once saturation is greater than $92 \%$. (A brief video of ADV is available online; see www.karger/com/doi/10.1159/000497776 for all online suppl. material). We consider abdominal surgery in the 4 weeks prior to bronchoscopy, and unstable fractures of the axial skeleton and/or long bones to be absolute contraindications to ADV. Incontinence and abdominal ostomies are relative contraindications.

For this study, each bronchoscopy was entered as a separate event, with a patient undergoing more than one bronchoscopy over the study interval entered once for each bronchoscopy. All hypoxic events over the study interval were recorded. For study purposes, a hypoxic event was defined as a saturation of less than $85 \%$ which lasted for $30 \mathrm{~s}$ or more. Transient desaturations of lesser degree and/or duration were not recorded. Chart review included amount of sedation used, need for ventilatory rescue, inability to complete procedures due either to inadequate sedation or to oversedation, and post-procedure need for higher level of care. For the last 6 months of the study interval, time from initiation of ADV to a saturation $>90 \%$ was available.

\section{Results}

Interventional pulmonary performed 893 bronchoscopies in the bronchoscopy suite over the study interval. Thirty-eight (4\%) of the bronchoscopies involved hypoxic events, thus triggering the hypoxemia protocol. Table 1 lists the characteristics of this study group. Mean patient age was 57 years (range, 18-95). Twenty (55\%) of the bronchoscopies were performed on men. Mean height was $172 \mathrm{~cm}$ (range, 149.4-198.1), and mean weight was 86 $\mathrm{kg}$ (range, 39.5-145.2). Twenty-five (68\%) of the procedures were performed on outpatients. 
The majority of bronchoscopies in the study group were endo-bronchial ultrasound procedures. Mean propofol dosing was $785 \mathrm{mg}$ (range, 150-1,010). Mean procedure time was 65 min (range, 35-95).

One of the 38 patients in the study group had a history of recent abdominal surgery for incarcerated hernia. Given this contraindication to ADV, he was ventilated with a bag/mask setup until hypoxia resolved. The remaining 37 patients were ventilated with ADV. In all 37 cases, the procedure was continued and completed to satisfaction without the need to remove the bronchoscope from the airways. For the subset for which the data were available $(n=11)$, mean time from initiation of ADV to a saturation of $90 \%$ was $17 \mathrm{~s}$, with a range of $10-27 \mathrm{~s}$. There were no post-procedural complaints of abdominal discomfort or any other new gastrointestinal symptoms. There was no escalation of care for any patient, and all outpatients were discharged after their procedures. Average time from arrival in recovery room to discharge was 34 min (range, 29-37).

\section{Discussion}

Prior to the emergence of positive pressure ventilation for ventilatory support in the 1960s and its subsequent dominance, three different approaches to ventilatory support had been developed; the iron lung, the rocking bed and the "pneumobelt" [5]. The latter two modalities effected ventilation by actively elevating the diaphragm via abdominal displacement and then allowing or assisting lung reexpansion as the abdominal contents moved caudally. The iron lung and its successor, the cuirass, used pressure variations to cause both abdominal and thoracic displacement. Most akin to the technique used in this study was the pneumobelt, a bladder belted over the abdomen that elevated the diaphragm with cyclic inflation and allowed the diaphragm to descend caudally during deflation $[5,6]$. The pneumobelt has no role in current acute care medicine but has been used for ventilatory support in patients with paralytic/restrictive respiratory insufficiency; in 1991, Bach and Alba demonstrated effective use of ADV in 48 patients for a mean of $12.9 \pm 11.5$ years (range, 2 months to 39 years) [7]. The one difference between prior experience and ours is that for sedation-induced hypoxia we have found an oscillatory rate of approximately 100 (similar to $\mathrm{CPR}$ rates) to be effective.

Positive pressure ventilation, both invasive and noninvasive, represents far the most common form of ventilatory assist used today. The only commonly used application of abdominal displacement today is the Heimlich maneuver, accepted as the optimal approach to asphyxiation due to aspiration because of its capacity to cause forceful expiratory flow. A cuirass ventilator, more akin to the iron lung than to the ADV modalities, is still commercially available, and there have been occasional case reports of cuirass ventilation during airway procedures, allowing unobstructed access to the upper airways [8, 9].

This is a report of consecutive cases of sedation-induced hypoxia and the application, when possible, of ADV. Hypoxia occurred in $4 \%$ of cases, and tended to occur when procedures were prolonged. (The average procedure duration and dosing of the study group far exceed overall averages for bronchoscopies performed by interventional pulmonary.) ADV was possible in 37 out of 38 consecutive cases of hypoxia. When utilized, ADV was uniformly effective. ADV reversed hypoxemia while allowing unencumbered access to the airways. In every case, the procedure was continued, and all relevant interventions were completed.

$\mathrm{ADV}$ is a simple intervention which requires no specific skills. There is one caveat; passive diaphragmatic descent is abetted when patients are upright or partially upright, as shown by Adamson et al. [6] For our procedures, patients are partially upright, and bronchoscopy is performed with the bronchoscopist standing to the right. Labs for which standard practice is 
to bronchoscope from the head with the patient completely supine $\left(0^{\circ}\right.$ elevation $)$ might find ADV to be less efficacious than it has been at this institution.

\section{Conclusions}

In summary, we describe an extended experience with ADV as a means of temporarily ventilating patients who have developed hypoxia during bronchoscopy. ADV is a simple, lowskilled intervention, which requires no special equipment. It is usually effective and, when effective, allows the bronchoscopist to continue and complete his/her procedure. There are no evident complications. ADV can be easily inserted into the approach to sedation-induced hypoxia. We hope that others will do so and will report their experiences.

\section{Statement of Ethics}

IRB \#228841 approval was sought and granted. The IRB also deemed this a non-human subject research, and thus no consent is required.

\section{Disclosure Statement}

Authors declare that they have no conflicts of interest pertaining to the data contained in this article.

\section{Funding Sources}

There was no funding used for this study.

\section{Author Contributions}

N.M. conceived, collected, and analyzed data. M.M. and J.D.C. assisted in data collection and analysis. All authors contributed to the written manuscript equally.

\section{References}

1 Bailey PL, Pace NL, Ashburn MA, Moll JW, East KA, Stanley TH. Frequent hypoxemia and apnea after sedation with midazolam and fentanyl. Anesthesiology. 1990 Nov;73(5):826-30.

2 Chhajed PN, Glanville AR. Management of hypoxemia during flexible bronchoscopy. Clin Chest Med. 2003 Sep; 24(3):511-6.

3 Stolz D, Kurer G, Meyer A, Chhajed PN, Pflimlin E, Strobel W, et al. Propofol versus combined sedation in flexible bronchoscopy: a randomised non-inferiority trial. Eur Respir J. 2009 Nov;34(5):1024-30.

4 Leiten EO, Martinsen EM, Bakke PS, Eagan TM, Grønseth R. Complications and discomfort of bronchoscopy: a systematic review. Eur Clin Respir J. 2016 Nov;3(1):33324.

5 Mehta S, Hill NS. Noninvasive ventilation. Am J Respir Crit Care Med. 2001 Feb;163(2):540-77.

6 Adamson JP, Lewis L, Stein JD. Application of abdominal pressure for artificial respiration. J Am Med Assoc. 1959 Apr;169(14):1613-7.

7 Bach JR, Alba AS. Intermittent abdominal pressure ventilator in a regimen of noninvasive ventilatory support. Chest. 1991 Mar;99(3):630-6.

8 Linton DM. Cuirass ventilation: a review and update. Crit Care Resusc. 2005 Mar;7(1):22-8.

9 Mori H, Shono A, Hirade R, Nikai T, Saito Y. Biphasic Cuirass Ventilation During Anesthesia for Tracheobronchial Stent Insertion or Removal by a Rigid Bronchoscope: A Case Report. A A Pract. 2018 Apr;10(8):198-200. 